This item was submitted to Loughborough's Research Repository by the author.

Items in Figshare are protected by copyright, with all rights reserved, unless otherwise indicated.

\title{
Submicrosecond pulsed atmospheric glow discharges sustained without dielectric barriers at kilohertz frequencies
}

PLEASE CITE THE PUBLISHED VERSION

PUBLISHER

(c) American Institute of Physics

\section{VERSION}

VoR (Version of Record)

\section{LICENCE}

CC BY-NC-ND 4.0

\section{REPOSITORY RECORD}

Walsh, James L., J.J. Shi, and Michael G. Kong. 2019. "Submicrosecond Pulsed Atmospheric Glow Discharges Sustained Without Dielectric Barriers at Kilohertz Frequencies". figshare. https://hdl.handle.net/2134/5181. 
This item was submitted to Loughborough's Institutional Repository (https://dspace.lboro.ac.uk/) by the author and is made available under the following Creative Commons Licence conditions.

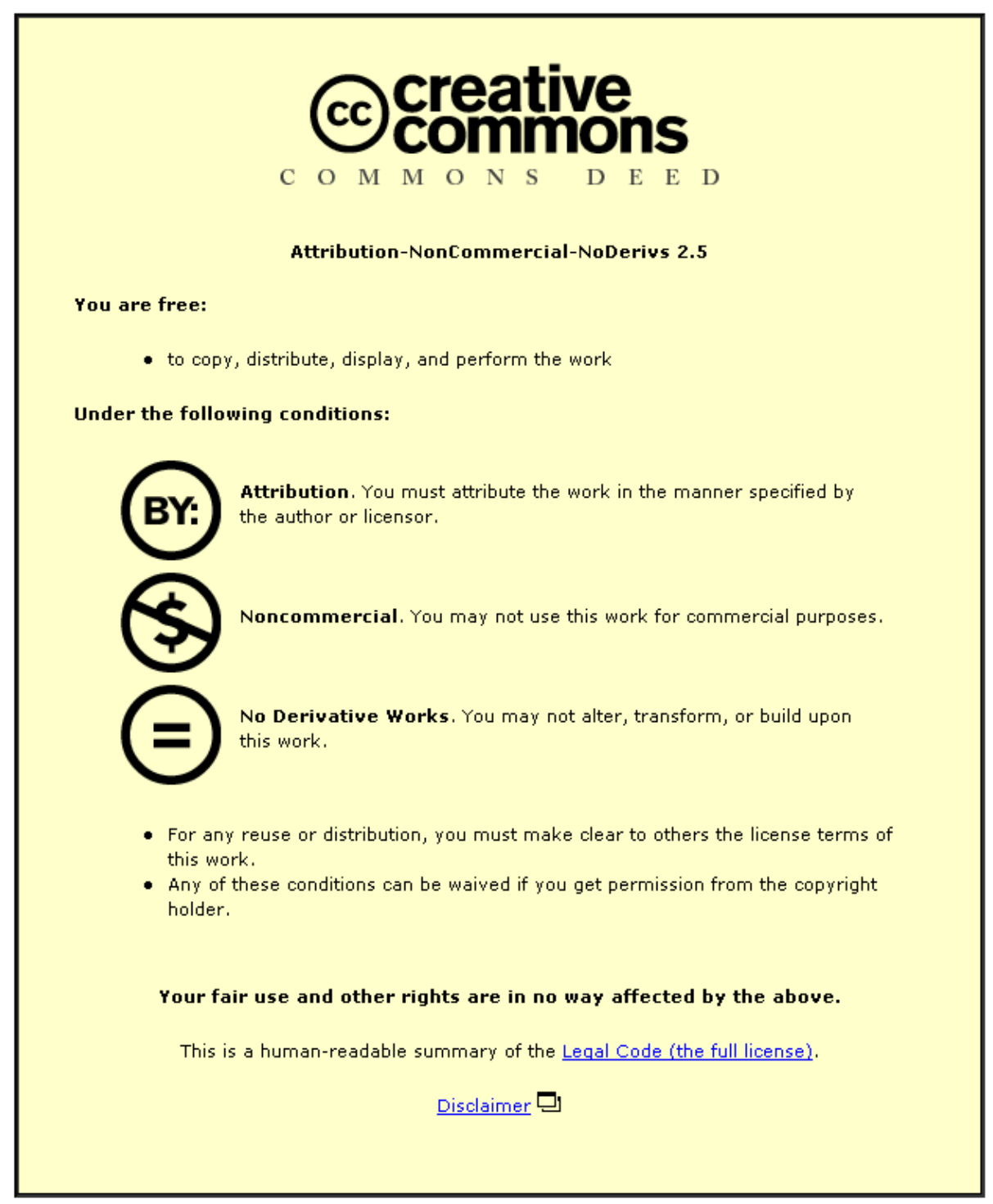

For the full text of this licence, please go to: http://creativecommons.org/licenses/by-nc-nd/2.5/ 


\title{
Submicrosecond pulsed atmospheric glow discharges sustained without dielectric barriers at kilohertz frequencies
}

\author{
J. L. Walsh, J. J. Shi, and M. G. Kong ${ }^{\text {a) }}$ \\ Department of Electronic and Electrical Engineering, Loughborough University, Loughborough, \\ Leicestershire LE11 3TU, United Kingdom
}

(Received 20 July 2006; accepted 31 August 2006; published online 19 October 2006)

\begin{abstract}
In this letter, the authors report the experimental observation of a large-volume atmospheric glow discharge sustained without dielectric barriers at $1 \mathrm{kHz}$. This barrier-free mode of operation is made possible with a submicrosecond pulsed excitation instead of the usual sinusoidal excitation. Its current-voltage characteristics are shown to be very different from conventional atmospheric dielectric barrier discharges, and its generation mechanism is studied with nanosecond resolved optical emission spectroscopy. The pulsed barrier-free atmospheric plasma is shown to produce very intense atomic oxygen emission line at $777 \mathrm{~nm}$, up to one magnitude more intensive than that of a comparable atmospheric dielectric barrier discharge. (C) 2006 American Institute of Physics.
\end{abstract}

[DOI: $10.1063 / 1.2361274]$

Atmospheric-pressure glow discharges (APGDs) offer a unique chamberless route to a wide range of highly desirable surface functionalities without wet chemistry and close to room temperature. ${ }^{1}$ In the excitation frequency range of $1-300 \mathrm{kHz}$, dielectric insulation of electrodes has long been believed to be essential for APGD generation. ${ }^{2}$ It is therefore of significant interest to study whether APGDs without dielectric barriers are fundamentally possible at kilohertz frequencies and, if so, whether barrier-free APGDs offer a different route to producing glow discharges at atmospheric pressure. From an application standpoint, it is sometimes desirable to dispense the use of the electrode-insulating dielectric layers that may become contaminated after continuous usage and so potentially lose their ability to stabilize APGD. So far, barrier-free APGD have been achieved largely outside the $1-300 \mathrm{kHz}$ range, either at the main frequency using effectively a large ballast resistor of various forms ${ }^{3,4}$ or at megahertz frequencies ${ }^{5-7}$ both to restrict current growth. Within the $1-300 \mathrm{kHz}$ range, the only reported barrier-free APGD is achieved by maintaining the sinusoidal applied voltage close to the gas breakdown voltage. ${ }^{8}$ In this letter, we report the experimental observation of a barrier-free APGD achieved with submicrosecond pulsed voltage instead of the sinusoidal excitation.

The submicrosecond pulsed barrier-free APGD employed two parallel stainless-steel electrodes with a surface area of $6 \mathrm{~cm}^{2}$ and a separation distance of $0.5-1.5 \mathrm{~cm}$. The two electrodes were naked without any dielectric barriers, and the electrode unit was housed within a Perspex box fed with a through flow of helium-oxygen mixture. The Perspex box was not airtight, and so oxygen and nitrogen were present. A home-made power source provided a train of unipolar voltage pulses of up to $4 \mathrm{kV}$ at $0.01-20 \mathrm{kHz}$. Figure 1 shows an example of a homogeneous APGD in an electrode gap of $1 \mathrm{~cm}$ and sustained with submicrosecond voltage pulses at $1 \mathrm{kHz}$. The image in Fig. 1(a) was taken with an exposure time of 1/60 s, whereas the image in Fig. 1(b) was taken in $10 \mathrm{~ns}$ with an Andor iCCD system (DH720). These

\footnotetext{
a) Author to whom correspondence should be addressed; electronic mail:
} m.g.kong@lboro.ac.uk clearly show a laterally uniform APGD without any streamers. The bottom electrode was the cathode and the bright thin layer near the cathode in Fig. 1(b) was the negative glow. Water cooling was not needed, and the electrode temperature measured with a thermocouple was found to be about $2{ }^{\circ} \mathrm{C}$ above room temperature. The generated plasma was stable and repetitive for many hours of continuous operation. These observations suggest that the pulsed barrier-free plasma was essentially a room-temperature, homogeneous, atmospheric glow discharge.

To understand the temporal behaviors of the submicrosecond pulsed APGD, we performed electrical measurements using a Tektronix oscilloscope (TDS 5054B) via wideband voltage and current probes. Figure 2(a) shows the currentvoltage characteristics of a barrier-free pulsed APGD in a $0.5 \mathrm{~cm}$ electrode gap and with a $\mathrm{He}-\mathrm{O}_{2}$ gas flow [He: 5 SLM (SLM denotes standard liters per minute) and $\mathrm{O}_{2}: 5$ SCCM (SCCM denotes cubic centimeter per minute at STP)]. The applied voltage was made of a train of unipolar $2 \mathrm{kV}$ pulses repeated at $1 \mathrm{kHz}$. Each voltage pulse had ap-
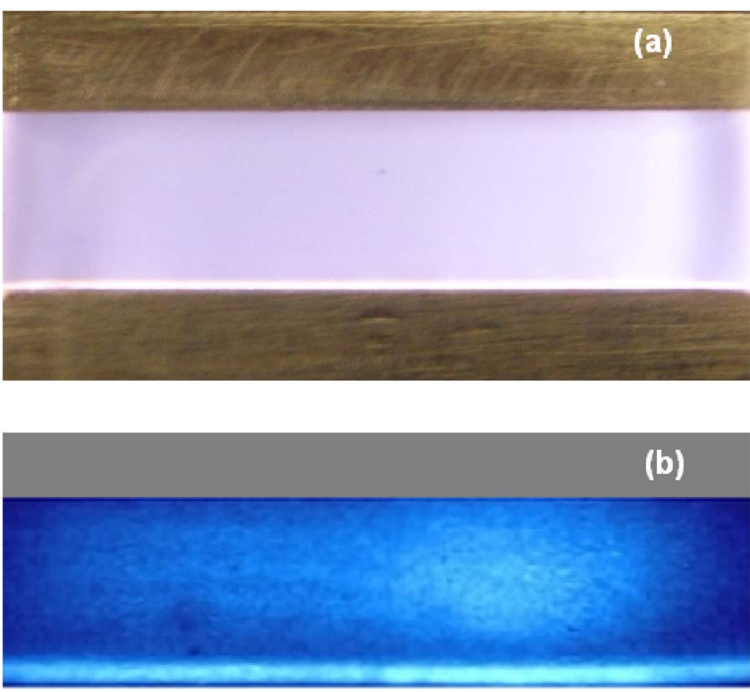

FIG. 1. (Color online) Images of a submicrosecond pulsed atmospheric glow discharge in a barrier-free helium gap of $1 \mathrm{~cm}$, taken with exposure times of (a) $1 / 60 \mathrm{~s}$ and (b) $10 \mathrm{~ns}$ 

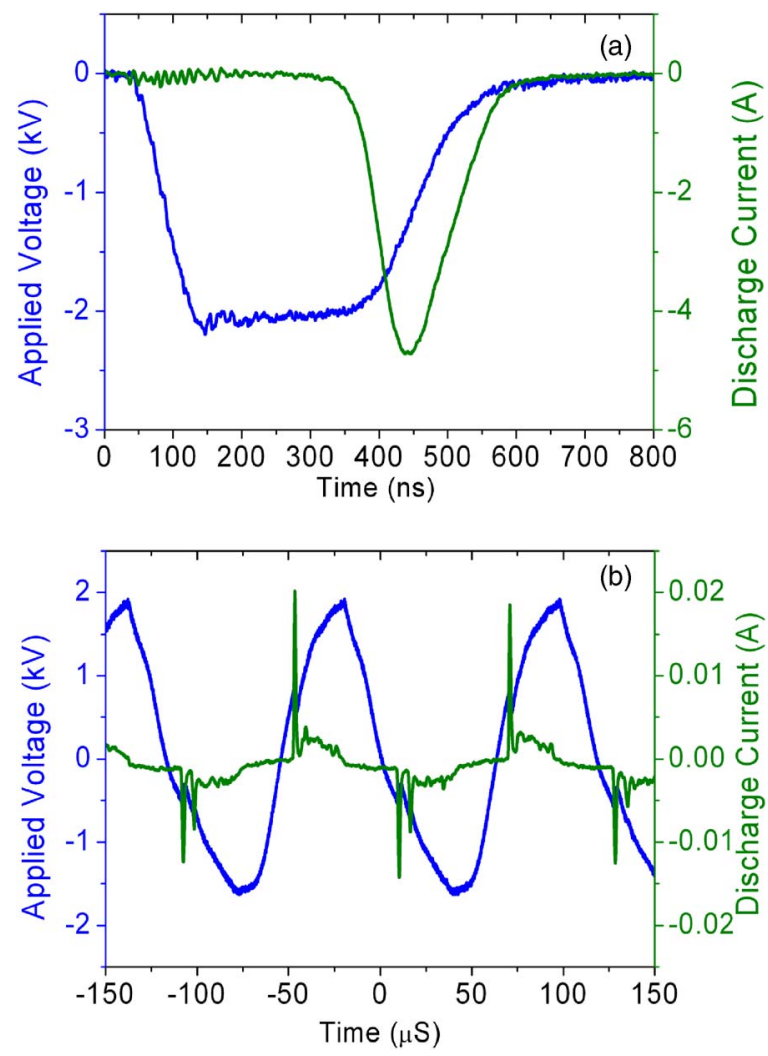

FIG. 2. (Color online) Current-voltage characteristics of (a) a $1 \mathrm{kHz}$ submicrosecond pulsed APGD and (b) an $8 \mathrm{kHz}$ atmospheric DBD, both generated with the same electrode unit and in an identical $\mathrm{He}-\mathrm{O}_{2}$ flow.

proximately a rise time of $100 \mathrm{~ns}$, a falling time of $200 \mathrm{~ns}$ (both between $10 \%$ and $90 \%$ of the peak voltage), and a pulse width of $360 \mathrm{~ns}$ (between two half-peak points). The duty cycle was very small at around $0.05 \%$. The discharge current was also periodic and its pulse width was about $120 \mathrm{~ns}$, markedly smaller than the voltage pulse width. Its peak was $4.84 \mathrm{~A}$ or $0.81 \mathrm{~A} / \mathrm{cm}^{2}$, much greater than that of atmospheric dielectric barrier discharges (DBDs) in $\mathrm{He}-\mathrm{O}_{2}$ mixture. $^{9}$

The pattern of a single current peak per cycle in Fig. 2(a) is also very different from that of conventional atmospheric DBD. ${ }^{9,10}$ Figure 2(b) shows current and voltage traces of a comparable atmospheric DBD produced with the same electrode unit, at the same peak voltage, and in the same gas mixture as that used for the pulsed APGD in Fig. 2(a). The excitation frequency was $8 \mathrm{kHz}$ since it was not possible to strike a stable DBD with our electrode unit at $1 \mathrm{kHz} .{ }^{11}$ Clearly, Fig. 2(b) shows the typical DBD pattern of one large current spike every half cycle. The cycle-to-cycle averaged peak current was $15 \mathrm{~mA}$ (or $2.6 \mathrm{~mA} / \mathrm{cm}^{2}$ ), about 323 times below that of the pulsed APGD in Fig. 2(a). The timeaveraged dissipated power density in the atmospheric DBD was $328 \mathrm{~mW} / \mathrm{cm}^{3}$, typical for atmospheric DBD. ${ }^{10}$ However, the averaged power density in the pulsed barrier-free APGD was about one magnitude lower at $38 \mathrm{~mW} / \mathrm{cm}^{3}$. We believe that the small duty cycle of the pulsed APGD was responsible for its smaller dissipated power even though its discharge current was much greater at the same applied voltage. Similarly, the pattern of one current peak per voltage pulse in Fig. 2(a) was different from that of pulsed atmospheric DBD in which two current peaks per voltage pulse were typical. ${ }^{12,13}$ These suggest that the pulsed barrier-free Downloaded 20 Aug 2009 to 158.125.80.71. Redistribution subject

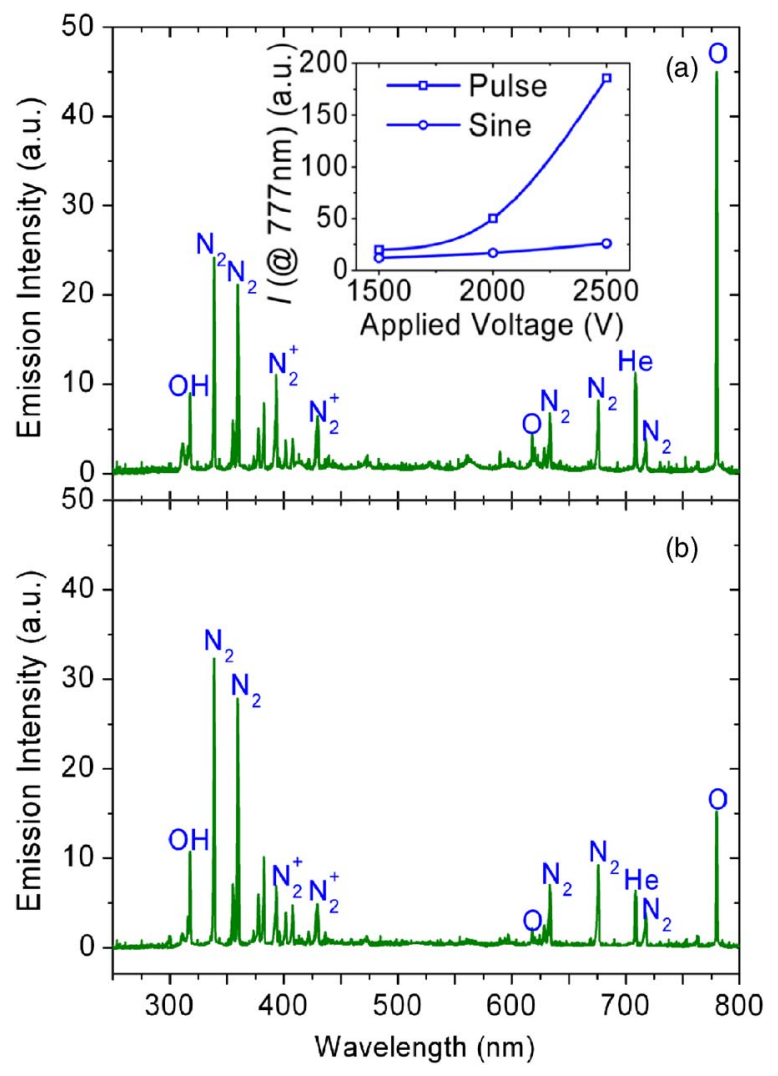

FIG. 3. (Color online) Optical emission spectra of (a) the pulsed barrier-free APGD of Fig. 2(a) and (b) the atmospheric DBD of Fig. 2(b), both at an applied voltage of $2 \mathrm{kV}$. The inserted diagram in (a) shows the optical intensity of oxygen atoms at $777 \mathrm{~nm}$ as a function of the applied voltage.

APGD in Fig. 1 was unlikely to be assisted by any dielectric barrier effect.

It is interesting to note that the current peak occurred in the falling phase of the applied voltage in Fig. 2(a). Suppose that we approximate the electron mobility in atmospheric $\mathrm{He}-\mathrm{O}_{2}$ mixture with that in atmospheric helium, $1132 \mathrm{~cm}^{2} \mathrm{~V}^{-1} \mathrm{~s}^{-1}$, and that the average electric field was $1 \mathrm{kV} / 0.5 \mathrm{~cm}=2 \mathrm{kV} / \mathrm{cm}$ in the voltage-rising phase. The time for an electron to transverse the electrode gap of $0.5 \mathrm{~cm}$ was about $221 \mathrm{~ns}$. Hence the voltage-rising phase of $100 \mathrm{~ns}$ was not sufficiently long for electrons in the electrode gap, if any, to reach the anode and support a large current in the external circuit. The displacement current made a similarly small contribution. With an electrode unit capacitance at $1.1 \mathrm{pF}$, the peak displacement current was $22 \mathrm{~mA}$ during the entire voltage pulse-228 times below the peak current of $4.84 \mathrm{~A}$ in Fig. 2(a). So the discharge during the voltage-rising phase was supported by a very small current and was at most a Townsend discharge. After the applied voltage reached its plateau, gas ionization continued and produced more electron-ion pairs. Eventually at $350 \mathrm{~ns}$, the discharge increased significantly and climbed to $4.84 \mathrm{~A}$ in about $100 \mathrm{~ns}$. Because of the very large current, the conductivity of the plasma load was likely to increase very significantly and as a result presented to the voltage source as an effective short circuit to cause the applied voltage to fall. Similar measurements were made with different voltage pulse widths and the current peak was always found to trigger the voltage fall.

Figure 3 shows the optical emission spectra of the pulsed barrier-free APGD and its corresponding atmospheric DBD, both with a peak applied voltage of $2 \mathrm{kV}$ and measured with to AIP license or copyright; see http://apl.aip.org/apl/copyright.jsp 


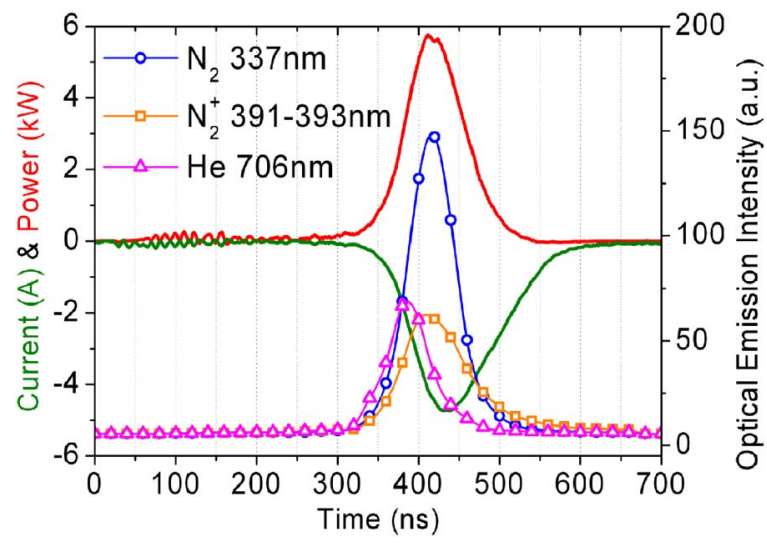

FIG. 4. (Color online) Nanosecond resolved optical signatures of three excited plasma species together with the current (green) and dissipated power (red) traces.

the same arrangement of an Andor Shamrock spectroscopy system at $1 \mathrm{~ms}$ exposure time. Both spectra exhibit spectral signatures of a very similar group of plasma species, for example, the dominant helium line at $706 \mathrm{~nm}$, the nitrogen ion line in $391-393 \mathrm{~nm}$ and the nitrogen line at $337 \mathrm{~nm}$, the $\mathrm{OH}$ (hydroxide) line at $309 \mathrm{~nm}$, and the atomic oxygen line at $777 \mathrm{~nm}$. Helium and oxygen lines were stronger in the pulsed APGD, and nitrogen lines were stronger in the atmospheric DBD. Oxygen atoms are useful as they are capable of modifying surface properties and inactivating bacteria. ${ }^{13-15}$ Figure 3 indicates that the atomic oxygen line in the pulsed APGD was about 3.7 times of that in the atmospheric DBD. This efficient production of oxygen atoms was found to improve further at larger applied voltages as shown in the insert in Fig. 3(a). As the applied voltage increased to $2.5 \mathrm{kV}$, the intensity of the $777 \mathrm{~nm}$ line became about one magnitude higher in the pulsed APGD than that in the atmospheric DBD. Hence the pulsed barrier-free APGD offers an energy-efficient route to the production of chemically reactive plasma species, such as atomic oxygen. Compared to sinusoidal barrier-free APGD, ${ }^{8}$ pulsed barrier-free APGD is also much more versatile, operated over a wider voltage range and at higher currents.

To further our understanding of the pulsed APGD, optical signatures of three excited plasma species were plotted in Fig. 4 together with the discharge current and dissipated power, all as a function of time on a nanosecond scale. Since the $\mathrm{N}_{2}^{+}$emission in the 391-393 nm range is the most intensive among ionic species in Fig. 3(a), its absence from the first $300 \mathrm{~ns}$ in Fig. 4 suggests the absence of significant amount of ions and hence electrons in the electrode gap. This is consistent with the fact that the current pulse became significant only after $300 \mathrm{~ns}$ even though the voltage pulse had persisted for the first $300 \mathrm{~ns}$ [see Fig. 2(a)]. Emission in $391-393 \mathrm{~nm}$ is known to be resulted from $\mathrm{N}_{2}^{+}\left(B^{2} \Sigma_{u}^{+}, v_{b}\right.$ $=0)$ transition to $\mathrm{N}_{2}^{+}\left(X^{2} \Sigma_{u}^{+}, v_{x}=0\right)$, and the former is populated through the Penning reaction and the charge transfer from $\mathrm{He}_{2}^{+}$ions. The temporal profile of the 391-393 nm line therefore reflects the evolution of the helium metastables and molecular ions. ${ }^{16}$ Similarly, the helium line at $706 \mathrm{~nm}$ indicates the presence of either energetic electrons or $\mathrm{He}_{2}^{+}$ions and low energy electrons. ${ }^{16}$ Again the absence of a strong $706 \mathrm{~nm}$ line before $300 \mathrm{~ns}$ supports the suggestion that there were insufficient electrons in the electrode gap to sustain a large current during the first $300 \mathrm{~ns}$ of the voltage pulse and that the discharge was at most a Townsend discharge.

It is worth noting that the temporal profiles of all three plasma species are similar. The instants at which the three emission lines reached their peaks were, however, different, and their fall times were also markedly different. For example, raw data suggest that the fall times of the 337 and $706 \mathrm{~nm}$ lines were 130 and $150 \mathrm{~ns}$, respectively. The ratio of their intensity integral over time in Fig. 4 was about 2, and compared well with the ratio of their time-accumulated intensity in Fig. 3. Difference in the temporal profiles of the three excited plasma species in Fig. 4 was probably related to their different life spans. It is also worth noting that their temporal profiles fit better with the power profile than the current profile. Given that the applied voltage tailed off during the current pulse in Fig. 2(a), it is possible that the average electron energy decreased in the latter phase of the current phase and the profile of the dissipated power offers a more accurate indication of the dynamics of energetic electrons. This suggests that the profiles of excited plasma species may be influenced by both their lifetimes and the dynamics of energetic electrons.

We have established that submicrosecond voltage pulses can be efficiently used to produce stable and homogenous APGD without dielectric barriers at kilohertz frequencies. Temporal behaviors of such barrier-free APGD were shown to be different from both sinusoidally excited and pulsed atmospheric DBDs. In addition, it has been demonstrated that they are a low-power source of high flux oxygen atoms.

This work was supported in part by the Department of Health (UK).

${ }^{1}$ J. R. Roth, Industrial Plasma Engineering: Principles (Institute of Physics, Philadelphia, 1995), Vol. 1, pp. 453-460.

${ }^{2}$ S. Kanazawa, M. Kogoma, T. Moriwaki, and S. Okazaki, J. Phys. D 21, 838 (1988).

${ }^{3}$ S. Okazaki, M. Kogoma, M. Uehara, and Y. Kimura, J. Phys. D 26, 889 (1993).

${ }^{4}$ M. Laroussi, I. Alexeff, J. P. Richardson, and F. F. Dyer, IEEE Trans. Plasma Sci. 30, 158 (2002).

${ }^{5}$ L. Baars-Hibbe, C. Schrader, P. Sichler, T. Cordes, K. H. Gericke, S. Buttgenbach, and S. Draeger, Vacuum 73, 327 (2004).

${ }^{6}$ J. J. Shi, X. T. Deng, R. Hall, J. D. Punnet, and M. G. Kong, J. Appl. Phys. 94, 6303 (2003).

${ }^{7}$ S. Y. Moon and W. Choe, Appl. Phys. Lett. 84, 188 (2004).

${ }^{8}$ J. J. Shi and M. G. Kong, Appl. Phys. Lett. 87, 201501 (2005).

${ }^{9}$ I. Radu, R. Bartnikas, and M. R. Wertheimer, IEEE Trans. Plasma Sci. 31, 411 (2003).

${ }^{10}$ M. G. Kong and X. T. Deng, IEEE Trans. Plasma Sci. 31, 7 (2003).

${ }^{11}$ X. T. Deng and M. G. Kong, IEEE Trans. Plasma Sci. 32, 1709 (2004).

${ }^{12}$ M. Laroussi, X. Liu, V. Kolobov, and R. Arslanbekov, J. Appl. Phys. 96, 3028 (2004).

${ }^{13}$ J. L. Walsh, J. J. Shi, and M. G. Kong, Appl. Phys. Lett. 88, 171501 (2006).

${ }^{14}$ M. Moravej, M. X. Yang, R. F. Hicks, J. Penelon, and S. E. Babayan, J. Appl. Phys. 99, 093395 (2006).

${ }^{15}$ X. T. Deng, J. J. Shi, G. Shama, and M. G. Kong, Appl. Phys. Lett. 87, 153901 (2006).

${ }^{16}$ G. Nersisyan and W. G. Graham, Plasma Sources Sci. Technol. 13, 582 (2004). 